\title{
A relação entre a utilização de cigarros eletrônicos e doenças pulmonares: uma revisão integrativa
}

\author{
The relationship between e-cigarette use and lung disease: an integrative review \\ La relación entre el uso de cigarrillos electrónicos y la enfermedad pulmonar: una revisión \\ integradora
}

Recebido: 21/12/2021 | Revisado: 29/12/2021 | Aceito: 02/01/2022 | Publicado: 03/01/2022

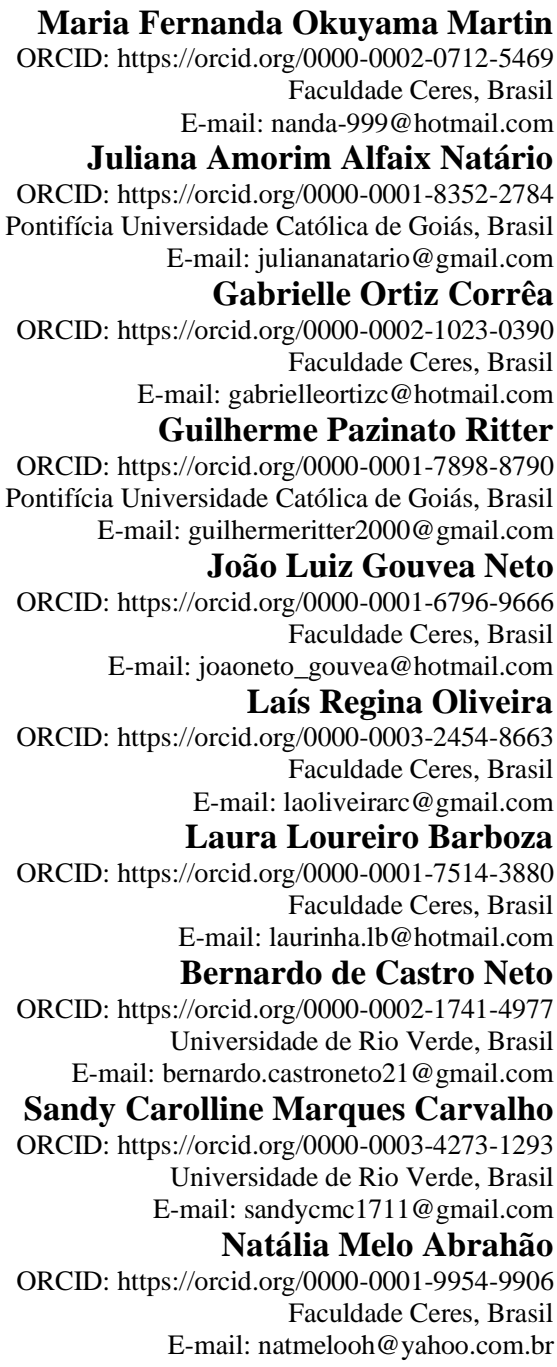

\section{Resumo}

Cigarros eletrônicos (CE) têm sido considerados por jovens e adultos, uma nova forma de lazer e ainda, uma alternativa para substituição dos cigarros convencionais e, por conta disso, têm chamado a atenção de diversas indústrias para sua fabricação. Porém, pouco se sabe, ainda, sobre seus reais malefícios. O objetivo deste trabalho foi descrever a relação entre o consumo de cigarros eletrônicos e as doenças pulmonares. O presente estudo apresenta-se como sendo uma revisão integrativa da literatura de natureza básica do tipo descritivo e qualitativo. Muitos autores acreditam que o cigarro eletrônico seja capaz de substituir os cigarros convencionais gradativamente, visto que seus malefícios são menores. Entretanto, outros cientistas acreditam que seus malefícios são diversos e que sua potencial nocividade é desconhecida, pois não se conhece a fundo ainda seus reais efeitos sob longa exposição e sua complexa estrutura, principalmente devido às inúmeras possibilidades na fabricação dos e-líquidos. Diversos autores já alertam sobre os riscos ao pulmão, principalmente com relação a EVALI e suas manifestações, acreditando que os CE podem inclusive, levar usuários a morte. Sob o ponto de vista lesgislativo, sua comercialização e propaganda ainda são 
proibidos. Mais pesquisas são necessárias para que se possa entender a fundo sobre suas consequências, limites da constituição do e-líquido, características do usuário e padtões e políticas de uso.

Palavras-chave: e-Cigarro; VAPE; Vapor do cigarro eletrônico; Doença pulmonar.

\begin{abstract}
Electronic cigarettes (EC) have been considered by young people and adults a new form of leisure and also an alternative to replace conventional cigarettes and, because of that, they have drawn the attention of several industries for their manufacture. However, little is known yet about its real harm. The aim of this work was to describe the relationship between the consumption of electronic cigarettes and lung diseases. The present study is presented as an integrative literature review of a basic descriptive and qualitative nature. Many authors believe that electronic cigarettes are able to gradually replace conventional cigarettes, as their harm is less. However, other scientists believe that its harms are diverse and that its potential harmfulness is unknown, as its real effects under exposure and its complex structure are not yet known in depth, mainly due to the possibilities in the manufacture of e-liquids. Several authors have already warned about the risks to the lung, especially in relation to EVALI and its manifestations, believing that FB can even lead users to death. From a legislative point of view, its marketing and advertising are still prohibited. More research is needed in order to understand the background about its consequences, limits of the constitution of the e-liquid, user characteristics and usage policies.
\end{abstract}

Keywords: e-Cigarette; VAPE; Electronic cigarette steam; Pulmonary disease.

\title{
Resumen
}

Los cigarrillos electrónicos (CE) han sido considerados por jóvenes y adultos una nueva forma de ocio y también una alternativa a la sustitución de los cigarrillos convencionales y, por ello, han llamado la atención de varias industrias por su fabricación. Sin embargo, aún se sabe poco sobre su daño real. El objetivo de este trabajo fue describir la relación entre el consumo de cigarrillos electrónicos y las enfermedades pulmonares. El presente estudio se presenta como una revisión integradora de la literatura de carácter descriptivo y cualitativo básico. Muchos autores creen que los cigarrillos electrónicos pueden reemplazar gradualmente a los cigarrillos convencionales, ya que su daño es menor. Sin embargo, otros científicos creen que sus daños son diversos y que se desconoce su potencial nocividad, ya que aún no se conocen en profundidad sus efectos reales bajo exposición y su compleja estructura, principalmente debido a las posibilidades en la fabricación de e-líquidos. Varios autores ya han advertido sobre los riesgos para el pulmón, especialmente en relación con EVALI y sus manifestaciones, creyendo que el FB puede incluso llevar a la muerte a los usuarios. Desde un punto de vista legislativo, su marketing y publicidad siguen estando prohibidos. Se necesita más investigación para comprender los antecedentes sobre sus consecuencias, los límites de la constitución del e-líquido, las características del usuario y las políticas de uso.

Palabras clave: Cigarrillo electrónico; VAPE; Vapor de cigarrillo electrónico; Enfermedad pulmonar.

\section{Introdução}

Cigarros eletrônicos (CE) (ou ainda dispositivos eletrônicos para fumar "DEF", e-cigarro ou vaporizador "VAPE") são cigarros constituídos de uma bateria de lítio, um sensor, um microprocessador, refil ou cartucho, uma solução líquida (também chamadas de e-líquidos), um atomizador responsável por aquecer e vaporizar esta solução líquida e um bocal para inalação. Por conta de sua composição, são conhecidos como cigarros mecânico-eletrônicos (Almeida-da-Silva et al., 2021; Grana et al., 2014).

Estes se assemelham aos cigarros convencionais tanto por conta de seu método de entrega de nicotina ao corpo quanto pela sua forma e, por conta disso, também são conhecidos como ENDS (electronic nicotine delivery systems). Tanto a nicotina quanto compostos carcinogêneos (como por exemplo, os carbonilos e as nitrosaminas específicas de tabaco) são substâncias que podem ser encontradas na maiorias dos vapores dos fumes, e portanto, são preocupantes não só para o pulmão quanto para todo o organismo (Hajek et al., 2020; Moheimani et al., 2017).

Além destes compostos, já citados anteriormente, o propileno glicol e o vapor de glicerol são componentes que podem ser encontrados em abundâncias nestes cartuchos de CE ou VAPE e são amplamente conhecidos por irritarem as vias aéreas superiores (Cao et al., 2020).

Por conta de todos estes fatores - e dos escassos estudos acerca de seus potenciais riscos à saúde humana, tanto direta quanto indiretamente a médio e longo prazo - este tema têm chamado a atenção de especialistas e cientistas de todo o mundo (Becker et al., 2021; Hess et al, 2016; Visser et al., 2019). 
Estudos dirigidos por Almeida et al. (2020, p. 7) que avaliaram 2.668 relatos de jovens e adultos hospitalizados com lesões pulmonares graves causadas pelo uso de CE (EVALI), apontam que as principais manifestações clínicas nestes pacientes foram: "dispneia (66,5\%), tosse (64\%), febre (60\%), fadiga (40\%) e dor torácica (35,5\%). Sintomas gastrointestinais foram frequentes: vômitos (48\%), náuseas (45\%), diarreia (25\%) e dor abdominal (19\%). Cerca 25\% dos pacientes apresentaram taquipneia e hipoxemia e 3,6\% hemoptise".

Uma revisão sistemática realizada por Tzorti et al. (2020) apontam que, analisando-se os relatos médicos acerca das alterações respiratórias causadas pelo uso de CE, a doença mais frequente foi a lesão pulmonar aguda seguida pela pneumonia, muito provavelmente associadas à exposição e inalação do vapor inserido no dispositivo.

Diante do exposto, o objetivo deste trabalho é descrever a relação entre o consumo de cigarros eletrônicos e as doenças pulmonares.

\section{Metodologia}

O presente estudo apresenta-se como sendo uma revisão integrativa da literatura. Sua natureza é básica, ou seja, pura ou fundamental. Ela existe para que se possa aumentar ou incrementar o conhecimento sobre um determinado assunto, ou seja, para que se possa focar nas melhorias de uma determinada teoria científica (Prodanov \& de Freitas, 2013).

Dentro de uma revisão integrativa, o papel do autor é buscar e avaliar criticamente e de forma resumida, as evidências que estão disponíveis para investigação de determinado tema, sendo seu resultado final uma ampla busca por estudos e por dados científicos. Neste tipo de pesquisa, admite-se um caráter descritivo do tipo qualitativo (Estrela, 2018).

Um estudo descritivo, por sua vez, tem como finalidade a descrição de um fato, hipótese ou evidência. Possui ainda, o objetivo de descrever de forma detalhada sobre o fenômeno que circunda a temática escolhida. Para que seja qualitativo, é fundamental que se analise os dados não numéricos (Prodanov \& de Freitas, 2013).

Os termos utilizados para a seleção das obras foram: ("cigarros eletrônicos") OR ("VAPE") OR (e-cigarrettes) AND ("doenças pulmonares"), inseridos na plataforma "Publish or Perish", tendo como bases de busca os seguintes: Google Scholar, PubMed e Web of Science, além de um recorte temporal de 2014 a 2021. Os idiomas admitidos, tanto para os descritores quanto para as obras foram: português, espanhol ou inglês. Os critérios de inclusão considerados neste estudo foram: artigos, livros ou trabalhos acadêmicos (de conclusão, dissertação ou tese), publicados entre os anos de 2014 e 2021, que apresentasse um dos descritores no título ou no resumo e que se apresentassem gratuitamente e na íntegra. Foram excluídos artigos de opinião, relatos de experiência e notas explicativas, artigos ou trabalhos acadêmicos duplicados entre as bases de busca, que não estivessem em uma das línguas acima mencionadas e artigos que não se apresentavam gratuitamente na íntegra.

Posteriormente, o download dos artigos foi realizado para que pudessem ser armazenados. Em seguida, os artigos e trabalhos acadêmicos encontrados foram minuciosamente analisados por 3 graduandos, para que pudesse ser verificado a íntima relação entre as obras e o tema abordado neste artigo. Posteriormente, os artigos foram organizados em uma planilha do Microsoft Excel® ${ }^{\circledR}$, considerando somente os artigos considerados "incluídos" neste artigo. A Figura 1 é a que melhor apresenta o detalhamento das obras, após aplicação dos critérios de inclusão e exclusão. 
Figura 1 - Representação do processo de seleção dos estudos resultantes da busca na plataforma Publish or Perish.

\begin{tabular}{|c|}
\hline $\begin{array}{c}\text { Encontraram-se } 68 \text { obras, após aplicação dos descritores: ("cigarros } \\
\text { eletrônicos") OR ("VAPE") OR (e-cigarrettes) AND (" doenças } \\
\text { pulmonares"), inseridos na pl ataforma "Publish or Perish", considerando o } \\
\text { recorte temporal de } 2014 \text { a } 2021\end{array}$ \\
\hline$\downarrow$ \\
\hline $\begin{array}{l}\text { Após a exclusão de artigos de opinião, relatos de experiência e notas } \\
\text { explicativas, alcançou-se o total de } 40 \text { obras }\end{array}$ \\
\hline$\downarrow$ \\
\hline Após exclusão dos duplicados, alcançou-se o total de 32 obras \\
\hline$\downarrow$ \\
\hline $\begin{array}{l}\text { Após a leitura crítica, } 5 \text { obras foram eliminados por não se adequarem ao } \\
\text { tema proposto }\end{array}$ \\
\hline$\downarrow$ \\
\hline $\begin{array}{c}\text { Um total de } 27 \text { obras ( } 24 \text { artigos, } 1 \text { livro e } 2 \text { trabalhos acadêmicos) foram } \\
\text { incluidos e utilizados nesta revisão }\end{array}$ \\
\hline
\end{tabular}

Fonte: Autores.

\section{Resultados e Discussão}

\subsection{As principais características dos cigarros eletrônicos e sua utilização no Brasil}

Cigarros eletrônicos vêm sendo amplamente utilizados como uma nova forma de lazer entre os jovens e adultos, bem como em substituição aos cigarros ditos "convencionais" (como o tabaco). Porém, embora muitos acreditem que seus efeitos nocivos sejam menores quando comparados ao tabaco, estudos demonstram que, dependendo de sua composição, este tipo de dispositivo pode ser considerado tóxico e até levar a morte (Knorst et al., 2014).

Os CE fornecem doses equivalentes ou maiores de nicotina (quando comparados ao tabaco) aos seus usuários (quando os fabricantes a utilizam em sua composição pois alguns não a incluem) além de ofertar alguns tipos de aditivos em aerosol. Em sua composição, podemos considerar principalmente três principais componentes: uma bateria recarregável, um atomizador e um cartucho contendo nicotina (Boléo-Tomé et al., 2019).

A estruturação do cartucho sofre oscilações, dependendo de cada tipo de fabricante. Em geral, são constituídos de nicotina e um componente específico para que se possa produzir o aerossol (como por exemplo: propilenoglicol, vapor de glicerol ou glicerol diluído em água). Estes componentes são considerados extremamente nocivos à saúde, podendo causar diversas intoxicações, alergias, irritações nas vias aéreas superiores, dentre outros tipos de reações (Cao et al., 2020; Menezes et al., 2021).

Para que se tornem mais atrativos para os jovens e adultos, muitos fabricantes costumam adicionar em seus cartuchos, compostos capazes de modificar o sabor, como por exemplo: extrato de baunilha, chocolate, menta, hortelã, café, frutas vermelhas, misturas específicas, etc. (Llambí et al., 2020). Existem, ainda, outras substâncias danosas que, com frequência, podem ser encontradas nestes tipos de dispositivos, como por exemplo: "formaldeído, acetaldeído, acroleína, compostos orgânicos voláteis, metais pesados e nitrosaminas derivadas do tabaco, foram identificadas nos cartuchos de nicotina" (Knorst et al., 2014, p. 565).

Dentre os constituintes do CE, a nicotina é a mais preocupante. Estudos demonstram que, além de ser considerada 
altamente estimulante, capaz de alterar o sistema nervoso central, acelerar os batimentos cardíacos e o metabolismo, aumentar a respiração e a pressão arterial, esta pode ser capaz de causar mais de 50 tipos de enfermidades, como por exemplo: alterações cardiovasculares, diversos tipos de cânceres e doenças pulmonares obstrutivas crônicas (DPOC). Por conta disso, diversos fabricantes apresentam o seu valor alterado em seus rótulos (muitas vezes de 16 a $24 \%$ inferior à quantificação real) (Drop et al., 2017; Simões, 2021).

Segundo Barufaldi et al. (2021) houve um aumento de 3\% de fumantes de CE ou DEF somente na pandemia, considerando a população de 18 anos ou mais. Estima-se que metade das pessoas que consomem ou já experimentaram este tipo de produto nunca foram consumidoras do cigarro convencional. Dados trazidos pelos mesmo autores afirmam que os usuários de cigarros eletrônicos correm quatro vezes mais risco de experimentação do cigarro convencional e quatro vezes mais risco de se tornarem tabagistas.

Considerando o perfil dos usuários, estima-se que em 2019, a prevalência da utilização de CE fosse de 0,64\% (porcentagem que representam, em média, 1 milhão de pessoas), das quais aproximadamente 70\% apresentavam entre 15 e 24 anos, $90 \%$ eram não fumantes e a maioria, eram habitantes da região Centro-Oeste do país. Dados também apontam que o uso de álcool e/ou narguilé estimulam a utilização de CE em mais de 80\% dos casos (Bertoni et al., 2021).

Além de todos os fatores acima citados, cabe ressaltar que os fumantes considerados passivos (isto é, que inalam a fumaça advinda dos fumantes passivos) também sofrem com a inalação dos vapores advindos do CE. Um estudo de revisão dirigido por Hess et al. (2016) afirma que, dos 16 artigos levantados, a maioria relata que os fumantes passivos afirmaram ter sua saúde afetada após exposição ao vapor advindo dos CE. Relataram, ainda, que a sua exposição pode ser ainda mais prejudicial e perigosa para crianças e bebês.

\subsection{Aspectos regulatórios relacionados a comercialização do CE}

Os CE e DEF são produzidos no Brasil desde o ano de 2003. Os primeiros rumores de sua concepção se deram quando tais produtos se apresentavam em formato de cigarros, porém mais longos, descartáveis, recarregáveis, com refis líquidos, em sistemas abertos ou fechados e que continham um dispositivo eletrônico capaz de acoplar um refil com tabaco, também conhecidos como "pods" (ANVISA, 2020).

Acredita-se que não haja segurança alguma em nenhum dos Dispositivos Eletrônicos para Fumar (DEF) que contenham nicotina - dentre outras substâncias químicas cientificamente não recomendadas para consumo e/ou ingestão - bem como não há nenhum documento oficial que demonstre seu potencial risco à saúde do usuário e nem sobre seus efeitos a curto, médio e longo prazo (Knorst et al., 2014).

Ainda que a fabricação e distribuição de tais produtos seja feita sob pouca ou nenhuma fiscalização até os dias atuais, tanto a comercialização quanto a importação e propagandas associadas a todos estes DEF são proibidas no Brasil, de acordo com a Resolução $n^{\circ}$ 46, de 28 de agosto de 2009. A justificativa de tal proibição pautou-se no fato de não haver dados científicos suficientes que comprovassem as alegações advindas dos fabricantes destes produtos (ANVISA, 2020).

As informações mais atuais sobre o tema é que Gerência-Geral de Registro e Fiscalização de Produtos Fumígenos está preparando um relatório de Análise de Impacto Regulatório (AIR) após aplicação, em 2019, de um Termo de Abertura do Processo Administrativo de Regulação (TAP) n 22. Este Termo representa o marco sobre as discussões acerca do "futuro" sobre os DEF. Seus debates, bem como uma possível conclusão acerca das decisões, estão previstos para serem concluídos no primeiro trimestre de 2022 (ANVISA, 2021).

\subsection{O que o cigarro eletrônico pode causar ao pulmão?}

Alguns autores relatam que os CE são menos prejudiciais à saúde (com menor probabilidade de causar câncer de 
pulmão, câncer oral ou doença cardíaca) quando comparados ao cigarro convencional pois não são capazes de ofertar ao usuário - e seus pulmões-, a imensa rede de substâncias químicas ao que só são originadas pela combustão (Franco et al., 2016; Pepper et al., 2015; Ralho et al., 2019).

Outros autores, como por exemplo Goniewicz et al. (2014) afirmam que os CE, apesar de parecerem inofensivos, são sim prejudiciais à saúde de seus consumidores pois os vapores gerados pelo dispositivo apresentam produtos tóxicos e nocivos, como por exemplo, a nicotina, metais pesados, compostos voláteis e agentes cancerígenos.

Para Kaur et al. (2018) embora presentes em quantidades menores, os aerossóis dos cigarros eletrônicos são conhecidos por conter muitos dos produtos químicos nocivos encontrados na fumaça do tabaco. Porém, acreditam que, embora tais evidências estejam claras, há ainda escassez de dados e evidências plausíveis que afirmem de forma conclusiva sobre os impactos toxicológicos, imunológicos e clínicos destes aerossóis aos pulmões.

Rosamonte et al. (2021, p. 40) afirmam que: "Os aerossóis contendo nicotina têm o potencial de afetar adversamente os mecanismos de defesa pulmonares (ex: comprometimento da função mucociliar e desregulação das respostas imunes de Th1 ao lipopolissacarídeo) podendo também estar associados a hipereatividade brônquica e desenvolvimento de doença pulmonar obstrutiva crônica (DPOC)".

Quando a sua composição é adulterada (pois há inúmeras combinações para o seu desenvolvimento e carregamento) os CE tornam-se mais perigosos. Por exemplo, quando inseridos de tetra-hidrocanabinol (THC) ou de suas modificações, as consequências podem se resumir a lesões pulmonares agudas ou ainda, graves (Santos et al., 2021).

Para Marques (2021) embora muitos autores acreditem que os efeitos nocivos dos CE sejam menores que os efeitos causados pelos cigarros convencionais, há inúmeras evidências entre o seu consumo e os processos fisiopatológicos de agressão e inflamação do epitélio respiratório. Além disso, acreditam que tais dispositivos sejam capazes de gerar partículas que, quando inaladas, geram estresse oxidativo além de inúmeros tipos de inflamações, metaplasia, desregulação de funções essenciais para a homeostasia pulmonar, como a produção e clearance do muco e resposta eficaz a agentes patogênicos.

Tzorti et al. (2020), em seus estudos acerca das "doenças e lesões relacionadas ao cigarro eletrônico: não apenas para o respiratório" apontam que, analisando-se os relatos médicos acerca das alterações respiratórias causadas pelo uso de CE, a doença mais frequente foi a lesão pulmonar aguda seguida pela pneumonia, muito provavelmente associadas à exposição e inalação do vapor inserido no dispositivo.

Dados trazidos por D'Almeida et al. (2020) afirmam que até janeiro de 2020 foram constatados 2.668 casos de EVALI que resultaram em hospitalização. Cento e sessenta e quatro pacientes que passaram por análise anatomopatológica apresentaram achados relacionados a: dano alveolar difuso, hemorragia alveolar; pneumonia lipoide, proteinose alveolar e bronquiolite obliterante com pneumonia em organização, em alguns casos. Até fevereiro de 2020, 68 levaram ao óbito.

Sabe-se que a exposição prolongada ao vapor e a utilização constante destes DEF podem resultar em complicações respiratórias, como asma, doença pulmonar obstrutiva crônica e inflamação (Kaur et al., 2018).

Estudos recentes apontam que os efeitos nocivos se estendem para além do pulmão, visto que a utilização de ecigarros podem acarretam em problemas cardiovasculares, imunológicos e de desenvolvimento neurológico. Os cientistas acreditam que os níveis em que estes produtos são capazes de afetar o ser humano, é dependente do tipo, da marca e da dose consumida (Overbeek et al., 2020).

Para Santos et al. (2021) devem haver mais estudos - tanto in vivo quanto in vitro - relacionados a utilização de DEF para que se possa correlacionar seus efeitos a síndrome de EVALI, pois há poucos relatos e resultados clínicos publicados sobre tema.

Em 2019, alertas foram enviados a médicos dos Estados Unidos a respeito de possíveis doenças pulmonares causadas por estes dispositivos, e então, foi disponibilizado um formulário eletrônico para que que se pudesse ter conhecimento das 
notificações e encaminhamentos a respeito de tais doenças (ANVISA, 2020).

\section{Considerações Finais}

Sabe-se que os cigarros eletrônicos são fabricados, distribuídos, comercializados e absorvidos pelo público jovem e adulto desde o ano de 2003. Seus três principais componentes são: uma bateria recarregável, um atomizador e um cartucho. Por serem fabricados de diferentes formas e modelos por diferentes tipos de indústrias, estes cartuchos podem conter diversas substâncias, como por exemplo: propilenoglicol, glicerina, água, nicotina e flavorizantes (responsáveis por ofertar sabor e aroma a estes produtos). Porém, o que se sabe é que algumas dessas substâncias são consideradas prejudiciais à saúde, de acordo com a sua forma e exposição.

Embora alguns autores defendam que a política de proibição da comercialização e do marketing de cigarros eletrônicos no Brasil afete a liberdade e a autonomia do indivíduo em consumi-los, o que se sabe é que no Brasil, desde 2009 a comercialização, importação e propaganda de qualquer DEF está terminantemente proibida.

A ANVISA divulgou diversas notas a respeito da abertura de uma espécie de "fórum" para que se pudesse debater a respeito de seu consumo, comercialização e propaganda, porém, até a presente data da submissão deste trabalho (dezembro/2020) o que se sabe é que não houve nenhuma liberação a respeito do tema.

A respeito dos possíveis malefícios e problemas causados pelos CE ou DEF ao pulmão, cientistas relatam que há diversas evidências de que a exposição e o contato com os vapores advindos destes dispositivos, tanto direta quanto indiretamente, são prejudiciais. Porém, embora alguns autores defendam a hipótese de que há dados suficientes que comprovem que o consumo de CE ou DEF afetem os processos fisiopatológicos de agressão e inflamação do epitélio respiratório, acarretem em inúmeros tipos de inflamações, metaplasia, desregulação de funções essenciais para a homeostasia pulmonar, como a produção e clearance do muco e resposta eficaz a agentes patogênicos, outros autores acreditam que tais efeitos não podem ser comprovados sem que se estude a completa e complexa composição dos cartuchos (ou seja, dos componentes químicos presentes e suas transformações), o tempo de exposição e a rotina de consumo destes produtos.

Acredita-se que há muito o que ser estudado e discutido, ainda, acerca deste tema. Sugere-se, como trabalhos futuros, que todas as substâncias adicionadas sejam amplamente e minuciosamente estudadas para que seus efeitos no organismo possam ser elucidados, bem como para que o seu consumo - e sua regularização - seja considerado tranquilo e seguro.

\section{Referências}

Agência Nacional de Vigilância Sanitária - ANVISA. (2021). Anvisa informa sobre processo regulatório de cigarros eletrônicos. www.gov.br. https://www.gov.br/anvisa/pt-br/assuntos/noticias-anvisa/2021/anvisa-informa-sobre-processo-regulatorio-de-cigarros-eletronicos.

Agência Nacional de Vigilância Sanitária - ANVISA. (2020). Cigarro eletrônico. www.gov.br. https://www.gov.br/anvisa/pt-br/assuntos/tabaco/cigarroeletronico.

Almeida-da-Silva, C. L. C., Dakafay, H. M., O'Brien, K., Montierth, D., Xiao, N., \& Ojcius, D. M. (2021). Effects of electronic cigarette aerosol exposure on oral and systemic health. biomedical journal, 44(3), 252-259.

Barufaldi, L. A., Guerra, R. L., Albuquerque, R. D. C. R. D., Nascimento, A. D., Chança, R. D., Souza, M. C. D., \& Almeida, L. M. D. (2021). Risco de iniciação ao tabagismo com o uso de cigarros eletrônicos: revisão sistemática e meta-análise. Ciência \& Saúde Coletiva, 26, 6089-6103.

Becker, T. D., Arnold, M. K., Ro, V., Martin, L., \& Rice, T. R. (2021). Systematic review of electronic cigarette use (vaping) and mental health comorbidity among adolescents and young adults. Nicotine and Tobacco Research, 23(3), 415-425.

Bertoni, N., Cavalcante, T. M., Souza, M. C. D., \& Szklo, A. S. (2021). Prevalência de uso de dispositivos eletrônicos para fumar e de uso de narguilé no Brasil: para onde estamos caminhando? Revista Brasileira de Epidemiologia, 24.

Boléo-Tomé, J. P., Pamplona, P., Rosa, P., \& Cordeiro, C. (2019). O Médico, o Doente Fumador e o Desafio dos Cigarros Eletrónicos. Acta Médica Portuguesa.

Cao, D. J., Aldy, K., Hsu, S., McGetrick, M., Verbeck, G., De Silva, I., \& Feng, S. Y. (2020). Review of health consequences of electronic cigarettes and the outbreak of electronic cigarette, or vaping, product use-associated lung injury. Journal of Medical Toxicology, 16(3), 295-310. 
D’Almeida, P. C. V., Silveira, M. B., Poiano, R., Américo, B., Padula, A. L., \& Santos, J. N. G. (2020). Lesóes Pulmonares Associadas ao Uso do Cigarro Eletrônico. Blucher, 6(4), 92-120.

Drope, J., Cahn, Z., Kennedy, R., Liber, A. C., Stoklosa, M., Henson, R., ... \& Drope, J. (2017). Key issues surrounding the health impacts of electronic nicotine delivery systems (ENDS) and other sources of nicotine. CA: a cancer journal for clinicians, 67(6), 449-471.

Franco, T., Trapasso, S., Puzzo, L., \& Allegra, E. (2016). Electronic cigarette: role in the primary prevention of oral cavity cancer. Clinical Medicine Insights: Ear, Nose and Throat, 9, CMENT-S40364.

Goniewicz, M. L., Knysak, J., Gawron, M., Kosmider, L., Sobczak, A., Kurek, J., \& Benowitz, N. (2014). Levels of selected carcinogens and toxicants in vapour from electronic cigarettes. Tobacco control, 23(2), 133-139.

Grana, R. A., Ling, P. M., Benowitz, N., \& Glantz, S. (2014). Electronic cigarettes. Circulation, 129(19), e490-e492.

Hajek, P., Pittaccio, K., Pesola, F., Myers Smith, K., Phillips-Waller, A., \& Przulj, D. (2020). Nicotine delivery and users' reactions to Juul compared with cigarettes and other e-cigarette products. Addiction, 115(6), 1141-1148.

Hess, I. M., Lachireddy, K., \& Capon, A. (2016). A systematic review of the health risks from passive exposure to electronic cigarette vapour. Public Health Res Pract, 26(2), e2621617.

Kaur, G., Pinkston, R., Mclemore, B., Dorsey, W. C., \& Batra, S. (2018). Immunological and toxicological risk assessment of e-cigarettes. European Respiratory Review, 27(147).

Knorst, M. M., Benedetto, I. G., Hoffmeister, M. C., \& Gazzana, M. B. (2014). Cigarro eletrônico: o novo cigarro do século 21? Jornal Brasileiro de Pneumologia, 40, 564-572.

Llambí, L., Rodríguez, D., Parodi, C., \& Soto, E. (2020). Cigarrillo electrónico y otros sistemas electrónicos de liberación de nicotina: revisión de evidencias sobre un tema controversial. Revista Médica del Uruguay, 36(1), 153-191.

Marques, M. D. C. (2021). Drogas fumadas e novas formas de fumar cigarros: o que se sabe sobre a relação com a doença pulmonar? (Masters dissertation). Universidade Beira Interior, Covilhã, Portugal.

Menezes, I. L., Sales, J. M., Azevedo, J. K. N., Junior, E. C. F., \& Marinho, S. A. (2021). Cigarro Eletrônico: Mocinho ou Vilão? Revista Estomatológica Herediana, 31(1), 28-36.

Moheimani, R. S., Bhetraratana, M., Peters, K. M., Yang, B. K., Yin, F., Gornbein, J., ... \& Middlekauff, H. R. (2017). Sympathomimetic effects of acute e-cigarette use: role of nicotine and non-nicotine constituents. Journal of the American Heart Association, 6(9), e006579.

Overbeek, D. L., Kass, A. P., Chiel, L. E., Boyer, E. W., \& Casey, A. M. (2020). A review of toxic effects of electronic cigarettes/vaping in adolescents and young adults. Critical Reviews in Toxicology, 50(6), 531-538.

Pepper, J. K., Emery, S. L., Ribisl, K. M., Rini, C. M., \& Brewer, N. T. (2015). How risky is it to use e-cigarettes? Smokers' beliefs about their health risks from using novel and traditional tobacco products. Journal of behavioral medicine, 38(2), 318-326.

Ralho, A., Coelho, A., Ribeiro, M., Paula, A., Amaro, I., Sousa, J., ... \& Carrilho, E. (2019). Effects of electronic cigarettes on oral cavity: a systematic review. Journal of Evidence Based Dental Practice, 19(4), 101318.

Rosamonte, S. João, L. \& Barros, R. (2021). Electronic cigarettes: what are their health effects? Revista de ciências da saúde da ESSCVP. 13(1).

Santos, M. O. P., Pimenta, A. S., da Costa, F. P. R., Ferrareto, N. S., Donato, R. S., \& Luchesi, B. M. (2021). Lesão pulmonar associada ao uso de cigarro eletrônico (evali): reflexões sobre a doença e implicações para as políticas públicas. Arquivos Catarinenses de Medicina, 50(2), 311-328.

Simões, S. D. A. (2021). Caracterização e quantificação por espectroscopia de nicotina em misturas líquidas para cigarros eletrónicos (Masters dissertation). Universidade do Algarve, Portugal.

Tzortzi, A., Kapetanstrataki, M., Evangelopoulou, V., \& Behrakis, P. (2020). A systematic literature review of e-cigarette-related illness and injury: not just for the respirologist. International journal of environmental research and public health, 17(7), 2248.

Visser, W. F., Klerx, W. N., Cremers, H. W., Ramlal, R., Schwillens, P. L., \& Talhout, R. (2019). The health risks of electronic cigarette use to bystanders. International journal of environmental research and public health, 16(9), 1525. 